Article

\title{
Prospective evaluation of circulating miR-126, mir-197 and mir- 223 in relation to cardiometabolic diseases
}

\author{
Widet Gallo 1,2,3, Filip Ottosson 1,2,3, Cecilia Kennbäck 4, Amra Jujic 1,5, Jonathan Lou S. Esguerra 2,3,6, Lena Eliasson \\ 2,3,6, Olle Melander 1,2,3,4*
}

1 Hypertension and Cardiovascular Disease, Department of Clinical Sciences-Malmö, Lund University, Malmö, 205 02, Sweden

2 Lund University Diabetes Centre, Department of Clinical sciences Malmö, Lund University Malmö, Sweden

3 Clinical Research Centre, Skane University Hospital, Lund and Malmö, Sweden

4 Department of Emergency and Internal Medicine, Skane University Hospital, Malmö, Sweden

5 Department of Cardiology, Skane University Hospital, Malmö, Sweden

6 Islet Cell Exocytosis, Department of Clinical Sciences-Malmö, Lund University, Malmö, , Sweden

* *Correspondence: widet.gallo@med.lu.se; Tel.: +46 40 391220; Department of Clinical Sciences-Malmö, Lund University, Clinical Research Centre, CRC, 91:12, Jan Waldenströmsgata 35, 21428 Malmö, Sweden

\begin{abstract}
We aim to investigate if serum levels of microRNAs: miR-126, mir-197 and mir-223, previously implicated in cardiometabolic disease, are reproducibly associated with incident-diabetes (inc-DM), incident-cardiovascular disease (inc-CVD) and with carotid atherosclerosis (measured for the maximum thickness of the intima-media of the carotid bulb (IMT)). The microRNAs were measured, one: in serum of 553 subjects from the baseline exam of the Swedish prospective cohort, Malmö Diet and Cancer Study (MDC-CC), with 169 subjects who developed CVD and 140 DM (16 years follow-up) and, two: in 1221 subjects from the Malmö Offspring Study (MOS), with 14 developed CVD and 12 DM (3.7 years follow-up). Multivariate logistic and linear regression models were used to investigate the relationship of serum-concentrations of the microRNAs and inc-DM, inc-CVD, IMT-bulb respectively. In MDC-CC, miR-126 showed significant positive association with inc-DM ( $p=0.01)$ whereas in fully adjusted model, the association was borderline significant $(p=0.05)$. The results were not replicated in MOS. There was no consistent significant association between the microRNAs with IMT or inc-CVD in any cohort. Our results do not support previous reports on significant associations between these microRNAs and the risk of CMD, as they were not reproducible in our cohorts. In addition, the directionality of any associations found were not consistent with those previously reported.
\end{abstract}

Keywords: MicroRNAs, miR-126, mir-197, mir-223, Cardiometabolic Disease, Diabetes, Cardiovascular disease, Atherosclerosis, Inter Media Thickness

\section{Introduction}

Diabetes mellitus, i.e., chronic hyperglycemia, occurs mainly due to defects in insulin secretion and insulin resistance [1,2]. Type 2 diabetes is one of the fastest growing diseases in the world and is considered as one of the major risk factors for cardiovascular disease (CVD) [3], which is mainly caused by atherosclerosis [4].

MicroRNAs are small non-coding RNAs, about 22 nucleotide-long, that regulate mRNA post-transcriptionally by interacting with the 3 '-untranslated regions (UTRs) of target mRNA and cause destabilization or degradation of mRNA [5]. They can be found in the circulation attached to e.g., RNA-binding proteins or in exosomes and vesicles [6].

As microRNAs in the circulation may represent both tissue leakage from paracrine signaling patterns and/or an endocrine signaling, they are attractive biomarkers for 
predicting different disorders, which has led to numerous studies in profiling of microRNAs to identify their relationship to new onset diabetes and cardiovascular disease [7-11].

In a small case-control study, Zampetaki et. al showed that mir-126 was associated with decreased risk for prevalent and incident diabetes [8] in the Brunek cohort. The finding in Zampetakis work was replicated in a Han Chinese study with a follow-up of 4 years, where they reported that miR-126 was decreased in 20 individuals with incident diabetes compared to healthy controls [10].

Further, in Brunek cohort, Zampetaki et. al investigated plasma level of miR-126 in 47 incident cases myocardial infarction (MI). In a multivariate model, consisting of miR126, miR-197, miR-223 and numerous risk factors, they showed that miR-126 was associated with an increased risk of myocardial infarction, whereas in the same model, both miR-197 and miR-223 were inversely associated to risk of MI [9].

Thus, in the literature regarding circulating micro-RNAs in relation to cardiometabolic disease, mir-126, mir-197 and mir-223 are currently the ones with most consistent results across different populations. However, there are discrepancies in the design and study size in these previous studies. Here, we set out to replicate the previous findings regarding association with cardiometabolic disease in two large and independent populationbased prospective cohorts. We thus aimed to evaluate the levels of circulating miR-126 in relation to incident diabetes (DM) in two cohorts, MDC-CC $(n=553)$ and MOS $(\mathrm{n}=1223)$. Further we aimed to evaluate the relationship between circulating levels of miR-126, miR-197 and miR-223 and incident CVD and carotid intima media thickness (IMT) in both cohorts.

\section{Materials and Methods}

\subsection{Subjects}

\subsubsection{Malmö Diet and Cancer -Cardiovascular Cohort (MDC-CC):}

MDC is a population-based prospective cohort where participants were examined in 1991-1996 ( $\mathrm{n}=28$ 449). The Malmö Diet and Cancer-Cardiovascular Cohort ( $\mathrm{n}=6103)$, aiming to study the epidemiology of carotid artery disease, is a sub-cohort of randomly selected participants from the main cohort, MDC. For this study, 553 subjects from the MDC-CC were selected. Those consisted of 259 controls, free from both prevalent and incident DM and CVD, 140 incident cases of DM and 169 incident cases of CVD during a follow-up of 16 years, as described earlier [7].

\subsubsection{Malmö Offspring Study (MOS):}

MOS is a family-based cohort study, initiated in 2013 in Malmö, Sweden. The participants of MOS consist of the children and grandchildren of the Malmö Diet and Cancer (MDC) as described elsewhere [12]. In this study, 1223 subjects were included, consisting of $>1200$ subjects (free from prevalent DM and CVD), of whom 12 incident DM and 14 incident CVD cases developed during a follow-up time of about 3 years. Endpoint retrieval has been described previously [13].

\subsection{MicroRNA Measurements}

The measurements of microRNAs have been described before [7]. In brief, $250 \mu$ l human serum was extracted using miRNeasy 96 Total RNA isolation kit (Qiagen) with some modifications. In MDC-CC, reverse transcription of microRNA-specific, stem-loop primers were used for cDNA synthesis, whereas in MOS, poly(A) tailing extension was used. Further information about cDNA synthesis and qPCR has been described before $[7,11]$. 
For data analysis and normalization for relative quantification of miRNA in MDC, geometric means of hsa-miR-17 and hsa-miR-106a was used as endogenous controls in the relative quantification using the $2-\Delta \Delta \mathrm{Ct}$ method [7]. For normalization of microRNAs in MOS, all Ct -values from selected samples were log-transformed and normalized against log-transformed hsa-miR-17-5p11.

\subsection{Statistics}

- Logistic regression was applied to calculate odds ratio (OR) and 95\% confidence intervals per one standard deviation increment of log-transformed levels of miR126 for incident diabetes in both MDC and MOS. In the first model, an adjustment for age and sex was applied, whereas in the second fully adjusted "model 2 " we adjusted for age, sex, body mass index (BMI), high density lipoprotein cholesterol (HDL) and triglycerides for both cohorts, except for insulin, which was available and thus included in MDC only.

- In MDC, logistic regression was applied to calculate ORs and 95\% confidence intervals per one standard deviation increment of log-transformed levels of miR126, miR-223 and miR-197, respectively and simultaneously, to incident CVD in different models.

- The first model was an age and sex adjusted model, the second one included age, sex, systolic blood pressure (SBP), antihypertensive therapy (ATH), current smoking status, low density lipoprotein cholesterol (LDL), HDL, DM status at baseline, triglycerides, BMI, and insulin. The third model included all covariates from the second model and additionally all three microRNAs (miR-126, miR-223 and miR-197) were entered simultaneously, as in the previous paper by Zampetaki et. Al [9]. mir-197 and insulin were not measured in MOS.

- Intima media thickness of the carotid bulb was measured using ultrasound as described in our previous paper [11]. Cross sectional relationship of miR-126, miR-223 and miR-197 and intima media thickness (IMT) in MDC was evaluated by applying 3 models. The first was age and sex adjusted model, the second fully adjusted linear regression (age, sex, SBP, AHT, current smokers, LDL, HDL, DM, Triglycerides, BMI, and insulin and the third one included all risk factors in the second model and the addition of all three microRNAs (miR-126, miR-223 and miR-197). In MOS miR-197 and insulin were not measured.

- All analytical tests were performed using IBM SPSS Statistics for Windows, version 26.0 (Armonk, New York, United States).

\section{Results}

- In MDC-CC, miR-126, miR-197 and miR-223 were measured in 553 individuals with a mean age of 59 years, with $51 \%$ female. In MOS, the corresponding number was 1223 individuals with a mean age of 42 years and 52\% female. Generally, and as expected due to the age difference and study design, the MDC cohort has more unfavourable risk factors than MOS, except for waist circumstance and fasting glucose, which are similar in both cohorts, as shown in Table 1. 


\begin{tabular}{|l|l|l|l|l|}
\hline \multicolumn{5}{l}{ Table 1. Baseline clinical characteristics of subjects in MDC-CC and MOS } \\
\hline & MDC-CC & MOS \\
\hline & $\mathrm{n}$ & mean (SD) & $\mathrm{n}$ & mean (SD) \\
\hline Age, y & 553 & $59.16 \pm 5.8$ & 1221 & $41.62 \pm 13.6$ \\
\hline Women, \% & 553 & 51.2 & 1221 & 51.8 \\
\hline BMI, kg/m² & 553 & $27.06 \pm 4.56$ & 1221 & $26.04 \pm 4.68$ \\
\hline Waist circumference, cm & 553 & $89 \pm 14$ & 1221 & $90 \pm 14$ \\
\hline Fasting glucose, mmol/L & 553 & $5.37 \pm 1.26$ & 1219 & $5.48 \pm 0.96$ \\
\hline LDL cholesterol, mmol/L & 553 & $4.27 \pm 0.92$ & 1217 & $3.21 \pm 1.02$ \\
\hline HDL cholesterol, mmol/L & 553 & $1.29 \pm 0.33$ & 1218 & $1.6 \pm 0.48$ \\
\hline Triglycerides, mmol/L & 553 & $1.47 \pm 0.70$ & 1208 & $1.17 \pm 0.70$ \\
\hline Systolic Blood Pressure, mmHG & 553 & $147.69 \pm 19.97$ & 1165 & $120.62 \pm 17.33$ \\
\hline Diastolic Blood Pressure, mmHG & 553 & $89.72 \pm 9.57$ & 1165 & $83.33 \pm 10.71$ \\
\hline
\end{tabular}

- In MDC-CC, age and sex adjusted logistic regression showed a significant positive association of miR-126 with incident DM, (OR per SD increment $=1.32$; $95 \%$ CI 1.07$1.63, p=0.01)$ whereas in the fully adjusted model, the association was borderline significant (OR per SD increment of miR-126 =1.26; $95 \%$ CI 1.00-1.66, p=0.05) as explained in Table 2 . The results were not replicated in MOS, neither in age and sex adjusted (OR per SD increment $=1.58 ; 95 \%$ CI 0.89-2.81, $\mathrm{p}=0.12$ ) nor in fully adjusted model (OR per SD increment =1.53; $95 \%$ CI 0.85-2.73, p=0.15), Table 2 .

Table 2. miR-126 and incident diabetes in MDC-CC and MOS

\begin{tabular}{|c|c|c|c|c|c|c|}
\hline \multirow{3}{*}{$\begin{array}{l}\text { OR }(95 \% \mathrm{CI}) \\
\text { (age and sex) } \\
\text { adjusted) }\end{array}$} & \multicolumn{3}{|c|}{ MDC-CC } & \multicolumn{3}{|c|}{ MOS } \\
\hline & $\begin{array}{l}\mathrm{N} / \mathrm{N} \\
\text { events }\end{array}$ & $\begin{array}{l}\text { Continuous analysis } \\
\text { (per SD increment) }\end{array}$ & P-value & $\begin{array}{l}\mathrm{N} / \mathrm{N} \\
\text { events }\end{array}$ & $\begin{array}{l}\text { Continuous analysis } \\
\text { (per SD increment) }\end{array}$ & P-value \\
\hline & $390 / 140$ & $1.32(1.07-1.63)$ & 0.01 & $1177 / 12$ & $1.58(0.89-2.81)$ & 0.12 \\
\hline $\begin{array}{c}\text { OR (95\% CI) } \\
\text { (fully adjusted) }\end{array}$ & $390 / 139 *$ & $1.26(1.00-1.66)$ & 0.05 & $1177 / 12$ & $1.53(0.85-2.73)^{* *}$ & 0.15 \\
\hline
\end{tabular}

* fully adjusted for Age, Sex, Body Mass Index, High Density Lipoprotein, Triglycerides, and Insulin

** fully adjusted for Age, Sex, Body Mass Index, High Density Lipoprotein and Triglycerides.

- In MDC, logistic regression showed no association between miR-126, miR-223, miR197 and CVD in any model, neither in age and sex adjusted nor in fully adjusted model. The lack of significance remained even when all three microRNAs were entered simultaneously (miR-126: OR per SD increment $=1.07 ; 95 \% \mathrm{Cl}=0.82-1.35, \mathrm{p}=$ 0.58, (miR-223: OR per SD increment $=1.08 ; 95 \% \mathrm{Cl}=0.88-1.32, \mathrm{p}=0.48),(\mathrm{miR}-197: \mathrm{OR}$ per $\mathrm{SD}$ increment $=1.14 ; 95 \% \mathrm{Cl}=0.90-1.45, \mathrm{p}=0.27$ ) as shown in Table 3 . 
Table 3. Risk Evaluation of three microRNAs in incident cardiovascular disease (CVD) and Intima Media Thickness (IMT) in MDC

\begin{tabular}{|c|c|c|c|c|c|c|}
\hline \multirow[b]{2}{*}{ microRNAs } & \multicolumn{3}{|c|}{ CVD } & \multicolumn{3}{|c|}{ IMT } \\
\hline & $\mathrm{N} / \mathrm{N}$ & OR $(95 \%$ CI $)$ & $P$-value & $\mathrm{N} / \mathrm{N}$ & Beta $(95 \% \mathrm{CI})$ & $P$-value \\
\hline \multirow{3}{*}{$\operatorname{miR}-126$} & $428 / 169^{*}$ & $1.12(0.92-1.36)$ & 0.25 & 401 & $0.08(0,002-0.13) *$ & 0.04 \\
\hline & $428 / 167^{a}$ & $1.13(0.92-1.38)$ & 0.27 & 399 & $0.06(-0.01-0.13)^{\mathrm{a}}$ & 0.08 \\
\hline & $428 / 167^{b}$ & 1.07 (0.82-1.35) & 0.58 & 399 & $0.08(0.01-0.15)^{b}$ & 0.02 \\
\hline \multirow{3}{*}{$\operatorname{miR}-223$} & $428 / 169^{*}$ & $1.03(0.85-1.25)$ & 0.73 & 401 & $0.06(-0,002-0.13)$ * & 0.06 \\
\hline & $428 / 167^{a}$ & $1.04(0.85-1.27)$ & 0.7 & 399 & $0.06\left(-0,003-0.13^{a}\right.$ & 0.06 \\
\hline & $428 / 167^{b}$ & $1.08(0.88-1.32)$ & 0.48 & 399 & $0,096(0.03-0.17)^{\mathrm{b}}$ & 0,007 \\
\hline \multirow{3}{*}{ miR-197 } & $428 / 169^{*}$ & $1.13(0.93-1.37)$ & 0.23 & 401 & $0.02(-0.04-0.09) *$ & 0.49 \\
\hline & $428 / 167^{a}$ & $1.17(0.95-1.45)$ & 0.15 & 399 & $0.02(-0.04-0.09)^{a}$ & 0.52 \\
\hline & $428 / 167^{b}$ & $1.14(0.90-1.45)$ & 0.27 & 399 & $0.03(-0.04-0.10)^{\mathrm{b}}$ & 0.35 \\
\hline
\end{tabular}

*Adjusted for age and sex.

a Adjusted for age, sex, systolic blood pressure, Anti-hypertensive treatment, current smoker, Low density lipoprotein, High density lipoprotein, Diabetes Mellitus, triglycerides, body mass index and insulin.

b Adjusted for age, sex, systolic blood pressure, Anti-hypertensive treatment, current smoker, Low density lipoprotein, High density lipoprotein, Diabetes Mellitus, triglycerides, body mass index, insulin, miR-126-3p, miR-197-3p and miR-223-3p.

- In MOS, logistic regression showed no association between miR-126 and miR-223 and CVD in any model, neither in age and sex adjusted (miR-126: OR per SD increment $=1.17 ; 95 \% \mathrm{Cl}=0.70-1.97, \mathrm{p}=0.54)$, (miR-223: OR per SD increment $=1.06 ; 95 \%$ $\mathrm{Cl}=0.61-1.82, \mathrm{p}=0.82$ ) nor in fully adjusted regression model adjusted (miR-126: OR per $\mathrm{SD}$ increment $=1.41 ; 95 \% \mathrm{Cl}=0.57-3.50, \mathrm{p}=0.46)$, (miR-223: OR per SD increment $=1.08 ; 95 \% \mathrm{Cl}=0.47-2.49, \mathrm{p}=0.85)$, Table 4 .

Since atherosclerosis is a major contributor to CVD, we tested whether there was a relationship between the current microRNAs and IMT, as measured at the baseline exam. 


\begin{tabular}{|c|c|c|c|c|c|c|}
\hline \multirow[b]{2}{*}{ MicroRNAs } & \multicolumn{3}{|c|}{ CVD } & \multicolumn{3}{|c|}{ IMT } \\
\hline & $\mathrm{N} / \mathrm{N}$ & OR (95\% CI) & $P$-value & $\mathrm{N} / \mathrm{N}$ & Beta $(95 \%$ CI) & $P$-value \\
\hline miR-126* & $1208 / 14$ & $1.17(0.70-1.97)$ & 0.54 & $1221 / 483$ & $0.01(-0.02-0.04)$ & 0.42 \\
\hline $\operatorname{miR}-126^{* *}$ & $1208 / 08$ & $1.41(0.57-3.50)$ & 0.46 & $1221 / 414$ & $0.02(-0.02-0.05)$ & 0.33 \\
\hline miR-223* & $1028 / 13$ & $1.06(0.61-1.82)$ & 0.82 & $1221 / 452$ & $0.01(-0.03-0.38)$ & 0.69 \\
\hline $\operatorname{miR}-223^{* *}$ & $1208 / 06$ & $1.08(0.47-2.49)$ & 0.85 & $1221 / 389$ & $-0.01(-0.04-0.03)$ & 0.64 \\
\hline
\end{tabular}

* Adjusted for age, sex.

** Adjusted for age, sex, systolic blood pressure, Anti-hypertensive treatment, current smoker, Low density lipoprotein, High density lipoprotein, Diabetes Mellitus, triglycerides, body mass index.

- In MDC, age and sex adjusted linear regression showed a significant positive association between (per SD increment) miR-126 and IMT ( $(=0.08,95 \% \mathrm{Cl}=0.002-0.13, \mathrm{p}=$ 0.04 ). No significant association was found in the fully adjusted model (๑ $=0.06,95 \%$ $\mathrm{Cl}=-0.01-0.13, \mathrm{p}=0.08$ ). In models simultaneously entering risk factors and all three microRNAs in MDC, we found significant positive associations between miR-126 and IMT $(\odot=0.08,95 \% \mathrm{Cl}=0.01-0.15, \mathrm{p}=0.02)$ and between miR-223 and IMT ( $(\mathrm{e}=0.10$, $95 \% \mathrm{Cl}=-0.03-0.17, \mathrm{p}=0.007)$. No association between miR-197 and IMT was shown, neither in age and sex adjusted nor in fully adjusted models (Table 3).

- In MOS, linear regression showed no significant association between (per SD increment) miR-126 and IMT ( $(=0.01,95 \% \mathrm{Cl}=-0.02-0.04, \mathrm{p}=0.42)$ adjusted for age and sex, neither in the fully adjusted model $(\odot=0.02,95 \% \mathrm{Cl}=-0.02-0.05, \mathrm{p}=0.33$ ). There was no association between miR-223 and IMT in age and sex adjusted model (๑) $=0.01,95 \%$ $\mathrm{Cl}=-0.03-0.38, \mathrm{p}=0.69)$ nor in the fully adjusted model $(\odot=-0.01,95 \% \mathrm{Cl}=-0.04-0.03, \mathrm{p}=$ 0.64) (Table 4).

\section{Discussion}

Previously, miR-126, miR-223 and miR-197 were reported to be involved in cardiometabolic disease [8,9]. Here we show that none of mir-126, mir-223 or mir-197 are reproducibly associated with new-onset cardiometabolic disease with the same directionality as previously reported, questioning the utility of these microRNAs as biomarkers and their possible pathophysiological role in cardiometabolic disease.

In our study, we showed miR-126 to be significantly positively associated with incident diabetes in MDC ( $\mathrm{n}=553)$, although the association attenuated and was borderline significant when additional risk factors were included. In MOS, the direction of the association was also positive but not statistically significant. However, our results are not concordant with previously mentioned findings, where miR-126 was negatively associated with incident diabetes [8].

None of the three investigated microRNAs, neither alone nor as multivariate combined, showed any association with incident CVD in any cohort. In the multivariate model, simultaneously entering risk factors and all three microRNAs, i.e., a similar model as applied in the study by Zampetaki et. al [9], significant association was found between mir-126, mir-223 and IMT in MDC-CC but not in MOS. We were thus not able to reproduce the previous findings, where miR-126 was associated with increased risk for myocardial infarction but only in the multivariate regression model simultaneously entering all three micro-RNAs [9]. Given the strong association previously reported between the three investigated microRNAs and cardiometabolic disease, it was surprising that none of the previous findings were replicated. To our best knowledge, this is the largest prospective study conducted, with two large population-based cohorts with high number of incident cases cardiometabolic disease in MDC-CC, although the number of incident cases in MOS were limited. Worth mentioning, we had additionally more than 
400 subjects with of IMT measured, a strong marker of subclinical atherosclerosis, in each cohort.

There are several differences in study design and methodology that potentially could explain some of the variation. For instance, in our study, we extracted microRNAs from serum, whereas plasma was used in the other studies. Serum has previously reported to have higher concentrations of microRNAs and has higher sensitivity and lower variability of microRNAs compared to plasma $[6,14]$. Also, we investigated only incident cases, unlike other studies where they also used prevalent to investigated. The reason why we focused on incident cases was that cases with prevalent disease may have effects on micro-RNAs from therapies given against DM or CVD, and their effects may obscure associations with disease susceptibility, which is what we search for. Another point is that the studies have different normalization strategies, that may lead to disparity. The chosen normalizer, to serve as reference gene(-s), should be stable and should have very low variation between tested microRNAs [15].

The strengths of our study are that we investigate the level of microRNAs in two larges prospective cohorts, MDC $(n=553)$ and MOS $(n=1223)$, in healthy people that developed cardiometabolic disease later. As serum has been reported to contain higher concentrations of microRNA and is more sensitive with less variability for microRNAs, we considered as the second strength $[14,16]$.

One limitation of our study is that the MOS cohort has fewer incident cases DM (12) and CVD (14) than MDC-CC with DM (140) and CVD (169) due to younger population and shorter follow-up time. An additional limitation is that we do not have serum levels of miR-197 in MOS because it was not included in the study design at the start and therefore was not measured.

\section{Conclusions}

It is a concern that the previously mentioned studies on microRNAs could not be replicated in two large and independent population-based cohorts. Our conclusion from this study is that we do not find support for the studied microRNAs to serve as biomarkers for prediction of cardiovascular diseases and diabetes. Future investigations should include broader panels of micro-RNAs in large cohorts and include replication in at least one independent cohort.

Author Contributions: Conceptualization, WG, JE and OM; Formal analysis, WG, FO and OM; Funding acquisition, LE and OM; Investigation, WG and OM; Project administration, JE and OM; Resources, CK and OM; Supervision, FO, JE and OM; Validation, WG and OM; Visualization, WG; Writing - original draft, WG; Writing - review \& editing, FO, CK, AJ, LE and OM.

Funding: This research was funded by This work was supported by the Swedish Foundation for Strategic Research (IRC LUDC), Swedish Research Council (SFO-EXODIAB), AIR Lund (Artificially Intelligent use of Registers at Lund University) research environment (VR; grant no. 201961406) and Lund University Infrastructure Grant 2020-2024. The project received grants from the Swedish Research Council (OM; LE (2019- 01406)); Region Skåne-ALF/ Skåne University Hospital (OM; LE), OM also received support from the Knut and Alice Wallenberg Foundation; the Göran Gustafsson Foundation; the Swedish Heart- and Lung Foundation, European Research Council ERC-AdG-2019-885003 and the Novo Nordisk Foundation. LE was also supported by The Swedish Diabetes Foundation (DIA2019- 545) and Diabetes Research \& Wellness Foundation. JLSE received funding from the European Union's Horizon 2020 Research and Innovation Programme under Grant Agreement No. 667191 (T2DSystems); Crafoord Foundation; Syskonen Svenssons foundation, and Albert Påhlsson Foundation (JLSE). FO was supported by the Ernhold Lundström Research Foundation, The Albert Påhlsson Foundation and The Hulda and E Condrad Mossfelt Foundation.

Institutional Review Board Statement: The study was approved by the Regional Ethics Committee in Lund, Lund University, Sweden (MOS: 2012/594 and MDC, MDC-CC: LU 51-90) and conducted in accordance with the Helsinki Declaration. 
Informed Consent Statement: Both written and oral informed consent was obtained from all patients.

Data Availability Statement: Supporting data will be made available upon request to the corresponding authors.

Acknowledgments: Not applicable

Conflicts of Interest: Not applicable

\section{References}

1. Reaven GM. Role of Insulin Resistance in Human Disease. Diabetes. 1988;37(12):1595-1607.

2. Liu Y, Gao G, Yang C, et al. The role of circulating microRNA-126 (miR-126): a novel biomarker for screening prediabetes and newly diagnosed type 2 diabetes mellitus. Int J Mol Sci. 2014;15(6):10567-10577.

3. Federation ID. IDF Diabetes Atlas, 9th edn. Brussels, Belgium: 2019. 2019.

4. Mendis S, Puska P, Norrving B, World Health O, World Heart F, World Stroke O. Global atlas on cardiovascular disease prevention and control / edited by: Shanthi Mendis ... [et al.]. In. Geneva: World Health Organization; 2011.

5. Bartel DP. MicroRNAs: target recognition and regulatory functions. Cell. 2009;136(2):215-233.

6. Weber JA, Baxter DH, Zhang S, et al. The microRNA spectrum in 12 body fluids. Clin Chem. 2010;56(11):1733-1741.

7. Gallo W, Esguerra JLS, Eliasson L, Melander O. miR-483-5p associates with obesity and insulin resistance and independently associates with new onset diabetes mellitus and cardiovascular disease. PLoS One. 2018;13(11):e0206974.

8. Zampetaki A, Kiechl S, Drozdov I, et al. Plasma microRNA profiling reveals loss of endothelial miR-126 and other microRNAs in type 2 diabetes. Circ Res. 2010;107(6):810-817.

9. Zampetaki A, Willeit P, Tilling L, et al. Prospective study on circulating MicroRNAs and risk of myocardial infarction. J Am Coll Cardiol. 2012;60(4):290-299.

10. Zhang T, Li L, Shang Q, Lv C, Wang C, Su B. Circulating miR-126 is a potential biomarker to predict the onset of type 2 diabetes mellitus in susceptible individuals. Biochem Biophys Res Commun. 2015;463(1-2):60-63.

11. Gallo W, Ottosson F, Kennbäck C, et al. Replication study reveals miR-483-5p as an important target in prevention of cardiometabolic disease. BMC Cardiovascular Disorders. 2021;21(1):162.

12. Brunkwall L, Jönsson D, Ericson U, et al. The Malmö Offspring Study (MOS): design, methods and first results. European Journal of Epidemiology. 2020.

13. Ludvigsson JF, Andersson E, Ekbom A, et al. External review and validation of the Swedish national inpatient register. BMC Public Health. 2011;11(1):450.

14. Mompeón A, Ortega-Paz L, Vidal-Gómez X, et al. Disparate miRNA expression in serum and plasma of patients with acute myocardial infarction: a systematic and paired comparative analysis. Scientific Reports. 2020;10(1):5373.

15. Marabita F, de Candia P, Torri A, Tegnér J, Abrignani S, Rossi RL. Normalization of circulating microRNA expression data obtained by quantitative real-time RT-PCR. Brief Bioinform. 2016;17(2):204-212.

16. Wang K, Yuan Y, Cho J-H, McClarty S, Baxter D, Galas DJ. Comparing the MicroRNA Spectrum between Serum and Plasma. PLOS ONE. 2012;7(7):e41561. 